\title{
On a nonlinear ordinary differential equation
}

\author{
by W. OKRASINSKI (Wrocław)
}

Abstract. A nonlinear differential equation of the form

$$
\left(k(u) u^{\prime}\right)^{\prime}=f(x) u^{\prime}
$$

is considered. Nonnegative solutions $u$ such that $u(0)=0$ and $u(x)>0$ for $x>0$ are studied. Some theorems about the existence and uniqueness of nonnegative solutions are given.

1. Introduction. For a description of the mathematical model of the infiltration of water the Boussinesq equation is used. This equation has the form

$$
\left(h h_{x}\right)_{x}=h_{t}
$$

in the one-dimensional case or

$$
\frac{1}{x}\left(x h h_{x}\right)_{x}=h_{t}
$$

in the radial case (see [3], [4], [5]). The unknown function $h$ denotes the height of the saturated region above the impervious base. With respect to applications, similarity solutions of (1.1) and (1.2) having the form

$$
h(x, t)=g\left(\frac{x}{r_{0}(t)}\right)
$$

are interesting. The function $r_{0}(t)$ denotes the reachness of the saturated region at the time $t$ (see [3], [4], [5]). In the case of the similarity solutions, equations (1.1) and (1.2) may be reduced to the ordinary differential equations

$$
\left(u u^{\prime}\right)^{\prime}=(1-x) u^{\prime} \quad(x \in\langle 0,1\rangle)
$$

AMS classification 34B15, 45G10.

$\mathrm{Key}$ words. Nonlinear ordinary differential equation, existence and uniqueness of nonnegative solutions. 
and

$$
\left(u u^{\prime}\right)^{\prime}=A^{-x} u^{\prime} \quad(A>1),
$$

where $u(x)=g(1-x)$ is a unknown function. From a physical point of view sufficiently smooth solutions $u$ of equations (1.1) and (1.2) satisfying conditions:

$$
u(0)=0 \quad \text { and } \quad u(x)>0 \quad \text { for } x \in\langle 0,1\rangle
$$

are interesting. In papers [4] and [5], the existence and uniqueness of nonnegative solutions of $\left(1.1^{\prime}\right)$ and $\left(1.2^{\prime}\right)$ were proved. The methods used were based on the special form of these equations and on the Banach fixed point theorem. In papers [1] and [2], the following equation was considered:

$$
\left(k(u) u^{\prime}\right)^{\prime}=(1-x) u^{\prime} .
$$

In this paper we shall investigate the nonnegative solutions of a more general equation than (1.5), namely

$$
\left(k(u) u^{\prime}\right)^{\prime}=f(x) u^{\prime} .
$$

From here we shall consider only equation (1.6). For the sake of simplicity we make the assumption that the equation may be considered on the nonnegative half-line $\overline{\boldsymbol{R}}_{+}=\langle 0,+\infty$ ). After a simple modification of assumptions the methods and results of this paper may also be applied in cases of $\left(1.1^{\prime}\right),\left(1.2^{\prime}\right)$ and (1.5).

2. Reduction of the differential equation to an integral one. Now we make more precise assumptions about functions $f$ and $k$. Let $\boldsymbol{R}_{+}=(0,+\infty)$. We suppose that

(i) $f: \overline{\boldsymbol{R}}_{+} \rightarrow \boldsymbol{R}_{+}$is a decreasing continuously differentiable function,

(ii) $k: \overline{\boldsymbol{R}}_{+} \rightarrow \overline{\boldsymbol{R}}_{+}$is a continuously differentiable function such that $k(0)=0$ and $k(x)>0$ for $x>0$.

Now we define a class $M_{0}$ of functions in which we shall study the solvability of equation (1.6).

Definition 2.1. A function $u: \overline{\boldsymbol{R}}_{+} \rightarrow \overline{\boldsymbol{R}}_{+}$belongs to the class $M_{0}$ if and only if $u \in C\left(\overline{\boldsymbol{R}}_{+}\right) \cap C_{2}\left(\boldsymbol{R}_{+}\right), u(0)=0, u(x)>0$ for $x>0$ and $\lim _{x \rightarrow 0^{+}} k(u(x)) u^{\prime}(x)=0$.

Now we can formulate the following theorem:

THeOREM 2.1. If $u \in M_{0}$ is a solution of (1.6), then $u$ satisfies the integral equation

$$
W(u(x))=\int_{0}^{x}\left[f(s)-f^{\prime}(s)(x-s)\right] u(s) d s
$$


where

$$
W(x)=\int_{0}^{x} k(s) d s .
$$

Proof. Integrating (1.6) from $\delta$ to $x(\delta>0)$, we obtain

$$
k(u(x)) u^{\prime}(x)-k(u(\delta)) u^{\prime}(\delta)=f(x) u(x)-f(\delta) u(\delta)-\int_{\delta}^{x} f^{\prime}(s) u(s) d s .
$$

Let $\delta \rightarrow 0^{+}$. We get

$$
k(u(x)) u^{\prime}(x)=f(x) u(x)-\int_{0}^{x} f^{\prime}(s) u(s) d s .
$$

Integrating (2.4) from 0 to $x$, we have

$$
W(u(x))=\int_{0}^{x}\left[f(s) u(s)-\int_{0}^{s} f^{\prime}(t) d t\right] d s .
$$

Integrating by parts the right-hand side of (2.5), we obtain (2.1).

Now we define a class $M$ of functions in which we shall consider the solvability of the integral equation (2.1).

Definition 2.2. A function $u: \overline{\boldsymbol{R}}_{+} \rightarrow \overline{\boldsymbol{R}}_{+}$belongs to the class $M$ if and only if $u \in C\left(\overline{\boldsymbol{R}}_{+}\right), u(0)=0$ and $u(x)>0$ for $x>0$.

We have $M_{0} \subset M$. Now we present the following theorem:

THEOREM 2.2. If $u \in M$ is a solution of (2.1), then $u$ belongs to $M_{0}$ and satisfies equation (1.6).

Proof. Let us note that the function $W$ defined by (2.2) is differentiable and strictly increasing on $\boldsymbol{R}_{+}$. Then (2.1) can be written as

$$
u(x)=W^{-1}\left(\int_{0}^{x}\left[f(s)-f^{\prime}(s)(x-s)\right] u(s) d s\right)
$$

where $W^{-1}$ denotes the inverse function to $W$. From (2.6) we infer $u^{\prime}(x)$ exists for $x>0$. We get

$$
u^{\prime}(x)=\left[f(x) u(x)-\int_{0}^{x} f^{\prime}(s) u(s) d s\right][k(u(x))]^{-1} .
$$

We have $u^{\prime} \in C\left(\boldsymbol{R}_{+}\right)$. Similarly we can show that $u^{\prime \prime}(x)$ exists and is continuous for $x>0$. From (2.7) we conclude that the function $u$ satisfies equality (2.4). From this we infer $\lim _{x \rightarrow 0^{+}} k(u(x)) u^{\prime}(x)=0$ and it is easy to see that $u$ satisfies equation (1.6). The theorem is proved.

Now we give some a priori estimates. 
THEOREM 2.3. If $u \in M$ is a solution of (2.1), then $u$ is a strictly increasing function satisfying the following a priori estimate

$$
V^{-1}\left(\prod_{0}^{x} f(s) d s\right) \leqslant u(x) \leqslant V^{-1}(f(0) x)
$$

where $V^{-1}$ is the inverse function to

$$
V(x)=\int_{0}^{x} \frac{k(s)}{s} d s .
$$

Proof. Since $u(x)$ is given by (2.7) and $f^{\prime}(x) \leqslant 0$ for $x \in \boldsymbol{R}_{+}$, we obtain $u^{\prime}(x)>0$ for $x>0$. Therefore, $u$ is a strictly increasing function. From (2.4) we get

$$
f(x) u(x) \leqslant k(u(x)) u^{\prime}(x) \leqslant\left[f(x)-\int_{0}^{x} f^{\prime}(s) d s\right] u(x)
$$

The right-hand side of $(2.10)$ is equal to $f(0) u(x)$. Dividing (2.10) by $u(x)$ and integrating from 0 to $x$, we get

$$
\int_{0}^{x} f(s) d s \leqslant V(u(x)) \leqslant f(0) x .
$$

From (2.11) we obtain (2.8).

From here we shall consider the existence and uniqueness of nonnegative solutions of the integral equation.

3. Solvability of the integral equation. In this section we shall study the existence of nonnegative solutions of the integral equation. We define the following integral operator

$$
T(p)(x)=W^{-1}\left(\int_{0}^{x}\left[f(s)-f^{\prime}(s)(x-s)\right] p(s) d s\right)
$$

for $p \in M$.

Remark 3.1. We have $T(p) \in M$.

Remark 3.2. If $p_{i} \in M(i=1,2)$ and $p_{1}(x) \leqslant p_{2}(x)$ for $x \in \overline{\boldsymbol{R}}_{+}$, then $T\left(p_{1}\right)(x) \leqslant T\left(p_{2}\right)(x)$ for $x \in \overline{\boldsymbol{R}}_{+}$.

Let

$$
\varphi(x)=V^{-1}\left(\int_{0}^{x} f(s) d s\right)
$$

and

$$
\bar{\varphi}(x)=V^{-1}(f(0) x)
$$


Remark 3.3. The functions $\underline{\varphi}$ and $\bar{\varphi}$ belong to $M$ and are strictly increasing.

Remark 3.4. We can write inequality $(2.8)$ as

$$
\underline{\varphi}(x) \leqslant u(x) \leqslant \bar{\varphi}(x) .
$$

We can show the following lemma:

Lemma 3.1. For $x \in \overline{\boldsymbol{R}}_{+}$

$$
T(\underline{\varphi)}(x) \geqslant \underline{\varphi}(x)
$$

and

$$
T(\bar{\varphi})(x) \leqslant \bar{\varphi}(x) .
$$

Proof. For the proof it is sufficient to prove the following equivalent inequalities:

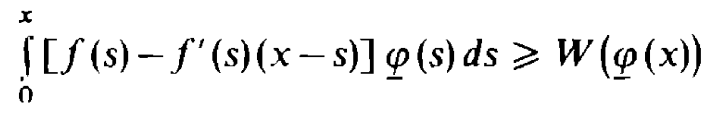

and

$$
\int_{0}^{x}\left[f(s)-f^{\prime}(s)(x-s)\right] \bar{\varphi}(s) d s \leqslant W(\bar{\varphi}(x)) .
$$

Let

$$
\Phi(x)=\int_{0}^{x}\left[f(s)-f^{\prime}(s)(x-s)\right] \underline{\varphi}(s) d s-W(\underline{\varphi}(x))
$$

and

$$
\Psi(x)=W(\bar{\varphi}(x))-\int_{0}^{x}\left[f(s)-f^{\prime}(s)(x-s)\right] \bar{\varphi}(s) d s .
$$

We have

$$
\Phi^{\prime}(x)=f(x) \underline{\varphi(x)}-\int_{0}^{x} f^{\prime}(s) \varphi(s) d s-k\left(\underline{\varphi(x))} \varphi^{\prime}(x)\right.
$$

and

$$
\Psi^{\prime}(x)=k(\bar{\varphi}(x)) \bar{\varphi}^{\prime}(x)-f(x) \bar{\varphi}(x)+\int_{0}^{x} f^{\prime}(s) \bar{\varphi}(s) d s
$$

But

$$
\underline{\varphi}^{\prime}(x)=\underline{\varphi(x)}\left[k(\underline{\varphi(x))}]^{-1} f(x)\right.
$$

and

$$
\bar{\varphi}^{\prime}(x)=\bar{\varphi}(x)[k(\bar{\varphi}(x))]^{-1} f(0) .
$$


From (3.9), (3.10), (3.11) and (3.12) we obtain

$$
\Phi^{\prime}(x)=-\int_{0}^{x} f^{\prime}(s) \underline{\varphi(s) d s}
$$

and

$$
\Psi^{\prime}(x)=-\int_{0}^{x} f^{\prime}(s)[\bar{\varphi}(x)-\bar{\varphi}(s)] d s .
$$

From (3.13) and (3.14) we infer $\Phi^{\prime}(x) \geqslant 0$ and $\Psi^{\prime}(x) \geqslant 0$ for $x \in R_{+}$. Since $\Phi(0)=0$ and $\Psi(0)=0$, we get $\Phi(x) \geqslant 0$ and $\Psi(x) \geqslant 0$. Hence inequalities (3.5) and (3.6) are true.

Now we present a theorem concerning the existence of solutions of (2.1) belonging to $M$.

TheOREM 3.1. Equation (2.1) has a solution belonging to $M$. $M$ :

Proof. We construct the following sequence of functions belonging to

$$
\begin{aligned}
\underline{v}_{0} & =\underline{\varphi}, \\
\underline{v}_{n+1} & =T\left(\underline{v}_{n}\right) \quad \text { for } n=0,1,2, \ldots
\end{aligned}
$$

Then, by Lemma 3.1, we have

$$
\underline{v}_{n+1} \geqslant \underline{v}_{n}
$$

and

$$
\underline{v}_{n} \leqslant \bar{\varphi} \quad \text { for } n=0,1,2, \ldots
$$

Then $\underline{v}_{n}(x)$ is convergent to the limit $\underline{u}(x)$ for all $x \in \overline{\boldsymbol{R}}_{+}$. By the Lebesgue theorem we obtain that $\underline{u}(x)$ satisfies equation (2.1) for all $x \in \overline{\boldsymbol{R}}_{+}$. Because of the properties of sequence (3.15) we get $\underline{u} \in M$.

Corollary 3.1. Equation (1.6) has a solution belonging to $M_{0}$.

This corollary follows immediately from Theorems 3.1 ans 2.2.

Remark 3.5. The sequence of functions

$$
\begin{aligned}
\bar{v}_{0} & =\bar{\varphi}, \\
\bar{v}_{n+1} & =T\left(\bar{v}_{n}\right) \quad \text { for } n=0,1,2, \ldots
\end{aligned}
$$

is convergent to a function $\bar{u}$ which belongs to $M$ and is a solution of (2.1).

Corollary 3.2. If $u \in M$ is a solution of (2.1), then

$$
\underline{u}(x) \leqslant u(x) \leqslant \bar{u}(x) \quad \text { for } x \in \overline{\boldsymbol{R}}_{+} .
$$

Proof of the corollary. From (3.4), (3.15) and (3.18) we have

$$
\underline{v}_{0}(x) \leqslant u(x) \leqslant \bar{v}_{0}(x) \quad \text { for } x \in \overline{\boldsymbol{R}}_{+} .
$$


By (3.15), (3.18) and (3.20) we get

$$
\underline{v}_{n}(x) \leqslant u(x) \leqslant \bar{v}_{n}(x) \quad \text { for } n=1,2, \ldots
$$

By the convergence of sequences $\underline{v}_{n}$ and $\bar{v}_{n}$ from (3.21) we obtain (3.19).

4. Uniqueness of nonnegative solutions. At first we show the local uniqueness of nonnegative solutions.

THEOREM 4.1. There exists an interval $\langle 0, \alpha\rangle(\alpha>0)$ such that $\underline{u} \equiv \vec{u}$ on $\langle 0, x\rangle$.

Proof. Let $u_{1}$ and $u_{2}$ denote, respectively, solutions $\underline{u}$ and $\bar{u}$. We have

$$
k\left(u_{i}(x)\right) u_{i}^{\prime}(x)=\int_{0}^{x} f(s) u_{i}^{\prime}(s) d s \quad(i=1,2) .
$$

By $w_{i}$ we denote the inverse function to $u_{i}(i=1,2)$. After the substitution $x=w_{i}(u)(i=1,2)$ we can write $(4.1)$ as

$$
k(u)\left[w_{i}^{\prime}(u)\right]^{-1}=\int_{0}^{u} f\left(w_{i}(s)\right) d s \quad(i=1,2) .
$$

From the last equality we get

$$
w_{i}(u)=\int_{0}^{u} k(s)\left[\int_{0}^{s} f\left(w_{i}(t)\right) d t\right]^{-1} d s
$$

Let $a>0$. We shall consider $w_{i}(u)(i=1,2)$ for $u \in\left\langle 0, u_{1}(a)\right\rangle$. We subtract $w_{1}$ and $w_{2}$. We obtain

$$
\begin{aligned}
& w_{1}(u)-w_{2}(u) \\
& =\int_{0}^{u} k(s)\left[\int_{0}^{s} f\left(w_{1}(t)\right) d t \int_{0}^{s} f\left(w_{2}(t)\right) d t\right]^{-1} \int_{0}^{s}\left[f\left(w_{2}(t)\right)-f\left(w_{1}(t)\right)\right] d t d s .
\end{aligned}
$$

From (4.3) we get

$$
\begin{aligned}
& w_{1}(u)-w_{2}(u) \\
& \leqslant\left[f\left(u_{1}(a)\right)\right]^{-2} \max _{s \in\langle 0, a\rangle}\left|f^{\prime}(s)\right| \max _{u \in\left\langle 0, u_{0}\right\rangle}\left(w_{1}(u)-w_{2}(u)\right) \int_{0}^{u} \frac{k(s)}{s} d s
\end{aligned}
$$

for $u \in\left\langle 0, u_{0}\right\rangle\left(u_{0} \in\left(0, u_{1}(a)\right\rangle\right)$. From (4.4) we get

$$
\begin{aligned}
\max _{u \in\left\langle 0, u_{0}\right\rangle} & \left(w_{1}(u)-w_{2}(u)\right) \\
& \leqslant\left[f\left(u_{1}(a)\right)\right]^{-2} \max _{s \in\langle 0, a\rangle}\left|f^{\prime}(s)\right| \int_{0}^{u_{0}} \frac{k(s)}{s} d s \max _{u \in\left\langle 0, u_{0}\right\rangle}\left(w_{1}(u)-w_{2}(u)\right) .
\end{aligned}
$$


Since $\int_{0}^{u} \frac{k(s)}{s} d s$ tends to zero as $x \rightarrow 0^{+}$, we can find $u_{0} \in\left(0, u_{1}(a)>\right.$ such that

$$
\int_{0}^{u_{0}} \frac{k(s)}{s} d s \ll\left[\max _{s \in\langle 0, a\rangle}\left|f^{\prime}(s)\right|\right]^{-1}\left[f\left(u_{1}(a)\right)\right]^{2} .
$$

From (4.5) we infer

$$
\max _{u \in\left\langle 0, u_{0}\right\rangle}\left(w_{1}(u)-w_{2}(u)\right)=0
$$

From (4.7) we get

$$
u_{1} \equiv u_{2} \quad \text { on }\langle 0, \alpha\rangle \text {, }
$$

where $\alpha=w_{2}\left(u_{0}\right)$.

Now we give the proof of the global uniqueness.

THEOREM 4.2. Equation (2.1) has the unique solution in the class $M$.

Proof. Let $u_{1}$ and $u_{2}$ denote, respectively, solutions $\underline{u}$ and $\bar{u}$. Let $b>\alpha$, where $\alpha$ is as in Theorem 4.1. Since

$$
u_{2}(x)-u_{1}(x)=W^{-1}\left(W\left(u_{2}(x)\right)\right)-W^{-1}\left(W\left(u_{1}(x)\right)\right),
$$

by the mean-value theorem we have the inequality

$$
u_{2}(x)-u_{1}(x) \leqslant \max _{s \in\left\langle W\left(u_{1}(\alpha)\right), W\left(u_{2}(b)\right)\right\rangle}\left[W^{-1}(s)\right]^{\prime}\left[W\left(u_{2}(x)\right)-W\left(u_{1}(x)\right)\right]
$$

for $x \in\langle 0, b\rangle$. From the last inequality we get

$$
\begin{aligned}
u_{2}(x)- & u_{1}(x) \\
& \leqslant\left[\min _{s \in\left\langle u_{1}(x), u_{2}(b)\right\rangle} k(s)\right]^{-1} \int_{0}^{x}\left[f(s)-f^{\prime}(s)(x-s)\right]\left[u_{2}(s)-u_{1}(s)\right] d s
\end{aligned}
$$

for $x \in\langle 0, b\rangle$. Let $B=f(0)+b \max _{s \in\langle 0, b\rangle}\left|f^{\prime}(s)\right|$ and $\beta>0$ be a number such that

$$
\left[\min _{s \in\left\langle u_{1}(a), u_{2}(b)\right\rangle} k(s)\right]^{-1} B \beta^{-1}<1 .
$$

Moreover, we define

$$
\varrho=\max _{s \in\langle 0, b\rangle} e^{-\beta s}\left(u_{2}(s)-u_{1}(s)\right)
$$

From (4.9) and (4.11) we get

$$
u_{2}(x)-u_{1}(x) \leqslant\left[\min _{s \in\left\langle u_{1}(\alpha), u_{2}(b)\right\rangle} k(s)\right]^{-1} B \beta^{-1} \varrho e^{\beta x}
$$

for $x \in\langle 0, b\rangle$. From (4.12) we obtain

$$
\varrho \leqslant\left[\min _{s \in\left\langle u_{1}(a), u_{2}(b)\right\rangle} k(s)\right]^{-1} B \beta^{-1} \varrho .
$$


By (4.10) we infer $\varrho \equiv 0$. The global uniqueness is proved.

Corollary 4.1. Equation (1.6) has the unique solution belonging to $M_{0}$.

\section{References}

[1] F. V. Atkinson, L. A. Peletier, Similarity profiles of flows through porous media, Arch. Rational Mech. Anal. 42 (1971), 369-379.

[2] - , - Similarity solutions of the nonlinear diffusion equation, ibidem 54 (1974), 373-392.

[3] J. Bear, D. Zaslavsky, S. I rmay, Physical principles of water percolation and seepage, UNESCO, 1968.

[4] J. Goncerzewicz, H. Marcinkowska, W. Okrasinski, K. Tabisz, On the percolation of water from a cylindrical reservoir into the surrounding soil, Zastosow. Mat. 16 (1978), $249-261$.

[5] W. Okrasiński, Integral equations methods in the theory of the water percolation. Mathematical methods in fluid mechanics, Proceedings of a conference Oberwolfach, 29.115.12.1981, Band 24, P. Lang. Frankfurt/M (1982), 167-176.

INSTITUTE OF MATHEMATICS

UNIVERSITY OF WROCI.AW

WROCLAW. POLAND

Reçu par la Rédaction le 13.01.1987 